\title{
Exploring Non-Aviation Information Sources for Aircraft Emergency Landing Planning
}

\author{
Pedro F. A. Di Donato* \\ National Civil Aviation Agency - ANAC, São José dos Campos, SP, 12242, Brazil \\ University of Michigan, Ann Arbor, MI, 48109, USA \\ Ella M. Atkins ${ }^{\dagger}$ \\ University of Michigan, Ann Arbor, MI, 48109, USA
}

\begin{abstract}
General aviation and small unmanned aircraft systems are less redundant, may be less thoroughly tested, and are flown at lower cruise altitudes than commercial aviation counterparts. These factors result in a higher probability of a forced or emergency landing scenario. Currently general aviation relies on the pilot to select a landing site even though workload in an emergency is typically high and decisions must be made rapidly. Existing moving map displays can highlight airport runways but provide little further guidance. This paper surveys the suite of emerging data sources in the context of forced landing site selection. Factors such as terrain, buildings, population, and winds must be holistically considered to select a safe off-runway site. Next, this paper investigates how one of these emerging databases, a cell phone occupation map, might be used to assist the pilot with forced landing site selection. One openly-available database is processed and analyzed to evaluate its utility and limitations.
\end{abstract}

\section{Nomenclature}

\author{
Acronyms \\ CDR \\ Call Detail Reports \\ GA \\ General Aviation \\ SMS \\ Short Message Service \\ UAS \\ Unmanned Aircraft System
}

$\begin{array}{ll}\text { Symbols } & \text { Mobile phone activity } \\ \phi & \text { Modified mobile phone activity for heat map } \\ \bar{\phi} & \text { Normalized mobile phone activity } \\ \hat{\phi} & \text { Proposed offline mobile phone activity metric } \\ \widetilde{\phi} & \text { Country code } \\ c & \text { Location, grid cell number } \\ g & \text { Total number of time intervals } \\ n_{t} & \text { Total number of country codes } \\ n_{c} & \text { Total number of grid tiles } \\ n_{g} & \text { 10-minute time interval initial time stamp, Unix Time }\end{array}$

*Regulation Specialist (on leave), PhD Student, Aerospace Engineering Department, Student Member.

${ }^{\dagger}$ Associate Professor, Aerospace Engineering Department, Associate Fellow. 


$\begin{array}{ll}\text { Subscripts } & \\ 1 & \text { SMS-In activity } \\ 2 & \text { SMS-Out activity } \\ 3 & \text { Call-In activity } \\ 4 & \text { Call-Out activity } \\ 5 & \text { Internet activity } \\ \max & \text { Maximum } \\ \min & \text { Minimum } \\ \text { avg } & \text { Average }\end{array}$

\section{Introduction}

Triply-redundant commercial transport aircraft are rarely forced to land off-runway, but emergency landings are occasionally required today in general aviation and experimental aircraft. Unmanned aircraft systems (UAS) will also require safe emergency landing capability prior to large-scale integration into the National Airspace System (NAS), particularly when operating over populated areas. General aviation (GA) and most UAS cannot afford triple-redundancy to substantially reduce the probability of emergency landing due to cost and weight considerations. GA and UAS operations also will not be cost-effectively should a team of two well-trained pilots be required to continuously supervise each flight, making inappropriate pilot/crew response also more likely for GA and UAS operations. GA and UAS applications therefore motivate further research in sensing, data mining, and software to reduce risks related to scenarios necessitating a forced or emergency landing.

Researchers have recently investigated the application of onboard sensors to identify potential landing sites, particularly for UAS. This solution strategy addresses a limiting case where no database link or previous knowledge of overflown terrain is available. Given knowledge of terrain and other risk-related quantities such as population density, aircraft decision aids can also take full advantage of database resources to minimize risk during an emergency landing.

Emerging information sources offer new opportunities to improve flight safety. For example, Automatic Dependent Surveillance-Broadcast (ADS-B) receivers are currently available to provide local air traffic data in support of detect-and-avoid (DAA) automation. While ADS-B is a significant advance, aviation need not remain insular with respect to data feeds. Quite generally, a cellular or wifi connection to onboard avionics opens a whole new world of information sources that can be exploited to improve aviation safety and to address new challenges that unmanned aviation will introduce.

Emergency landing is an important case where aviation is forced to interact with the "non-aviation" world. If landing on a conventional runway or helipad is not a feasible option, pilots must now choose a landing site based on prior knowledge of nearby terrain and at best a hurried examination of charts. Slowflying UAS can use sensors to inspect a local area for landing sites in real-time, but safe sites may not exist within onboard sensor range. Aircraft with access to maps as well as real-time data describing overflown terrain could potentially provide a much safer solution.

This paper investigates infusion of non-traditional data sources to better inform emergency landing site selection and planning. In previous work, the authors began to explore this idea through incorporation of a road database to identify potential off-field emergency landing sites 1 This new work first presents an overview of current information sources proposed in the literature for emergency landing planning. It further expands previously-considered data sources by proposing the use of mobile phone locations to track region occupancy or real-time population densities. Mobile phone usage data could be collected in real-time or using historical data to identify areas prone to high occupancy. As a case study, a cellular service provider's open database is processed to extract pertinent information. Benefits and limitations of the examined database are analyzed.

\section{Data Sources and Interfaces}

Figure 1 highlights onboard and ground-based or "cloud-based" subsystems that can collectively contribute to safe aircraft emergency landings. A variety of scenarios might necessitate an emergency landing, e.g., low fuel or stored battery energy, propulsion system failure resulting in reduced or loss of thrust, struc- 
tural or control surface damage. The operating or performance envelope will certainly be specific to the encountered situation, but the requirement to select a viable emergency landing site is common to all.

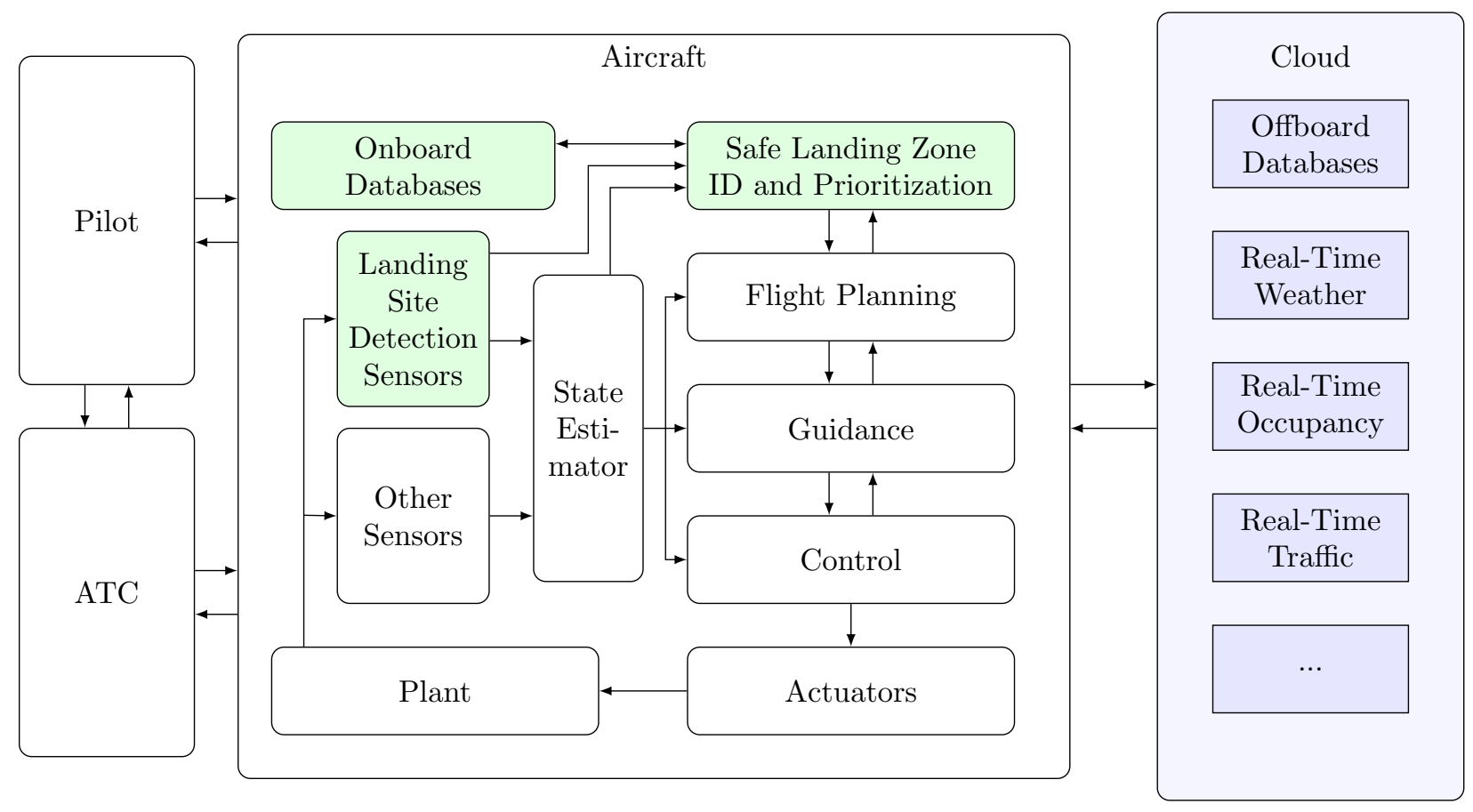

Figure 1. Aircraft Emergency Landing Components and Interfaces.

Figure 1 includes Flight Planning, Guidance and Control blocks that represent a standard autopilot control architecture to drive the Actuators of aircraft Plant. Once a goal landing site is identified and a flight plan to that site is generated, the pilot or autopilot guidance and control system executes this plan. Typically flight path angle is constrained but directional (lateral) flight control is not constrained in heading but is constrained by turn radius and rate. Steering a damaged aircraft to a landing site may require an adaptive or damage-case-specific flight control law. The problem of successfully executing a fully-autonomous emergency landing has been studied in previous work $!^{2}$

The Other Sensors block includes traditional inertial measurement unit, global positioning system (GPS), and air data sensors used for guidance, navigation, and control. Although Figure 1 differentiates these from specialized sensors used to identify potential landing sites such as cameras and lidar, information from both sources is available to the State Estimator to provide feedback for landing site selection or "Safe Landing Zone ID and Prioritization" as well as traditional flight planning, guidance and control.

Safe landing zone identification using onboard sensors is explored in the literature ${ }^{2 / 4}$ Typically cameras are used for this task, with human operators and/or computer vision systems used to identify safe or bestavailable landing sites within the camera field of view. Some work involves identifying and guiding the aircraft to a known and potentially marked landing area; other work identifies and navigates to a new site based strictly on natural visual cues!

Most sensor-based approaches do not exploit database information describing terrain or other pertinent site characteristics. Ref. 3 uses Digital Elevation Maps (DEM) for slope evaluation at a previously-selected candidate landing zone. Terrain data and real-time vision information are fused to identify potential landing sites in Ref. 4. A quadcopter emergency landing planner use terrain, urban population, and building databases to identify a path to a region or zone likely to provide a safe landing site in Ref. 5. Use of a road database to identify possible general aviation landing zones was proposed by the authors in prior work 1

When multiple landing sites are identified, they must be ranked to guide the automation or pilot in final selection of a landing site. Range to the site is a straightforward method of ranking, but this may not be the best choice particularly when the aircraft is not placed at greater risk by flying somewhat longer or farther. More sophisticated metrics combine attributes over landing site and enroute risk factors. For example, UAS landing sites can be ranked from multiple objective optimization ${ }^{3}$ or safety scores that account for models of uncertainty ${ }^{4}$. Diverse landing site selection and route planning metrics have also been proposed for manned 
aviation $\left[\begin{array}{l|l|l|l|}1 & 6 & 7 \\ \hline\end{array}\right.$

Air traffic control (ATC) can also offer new support for emergency landing. Currently, human pilots are asked to declare an emergency and their intentions, while ATC is responsible for clearing the airspace and any runway selected by the pilot for landing. However, with this process the human crew shoulders the full burden of identifying a landing site and flying there. NASA recently proposed a UAS Traffic Management $\left(\mathrm{UTM}^{\sqrt{8}}\right.$ prototype that provides support for UAS contingency management, specifically to help a UAS find an appropriate and safe landing area in case of an emergency situation. A proposed approach to air traffic control for low-altitude UAS includes a provision for operators to coordinate with emergency response resources in case of a crashed UAS,, 9 but this work does not discuss in detail specific support such coordination could provide. Note that Ref. 9 focuses on traditional aviation information sources such as radar, weather radar and lightning detection.

In recent years aviation, along with the rest of the world, has experienced the introduction of third party information service providers as shown above in Figure 1 in the Cloud module. The Federal Aviation Administration (FAA) uses the term Flight Information Service Data Link (FISDL) to describe these providers $\frac{10}{10}$ Perhaps the best example for general aviation is weather services provided by satellite radio which are reported to already provide data products under consideration a certifiable data source by the FAA 11

Exploitation of information sources beyond the traditional aviation datasets has been recently proposed by others as in Ref. 12. The authors' goal was to identify data from the "Internet of Things" to support dynamic risk assessment for manned aircraft traffic management. This work proposed integration of live flight information from ADS-B and live weather data with information from a terrain database, population database, and aircraft performance and noise models. Terrain and population databases, although theoretically dynamic, are rarely updated due to the fact that their records do not rapidly evolve, similar to the road database previously used by the authors in Ref. 1 .

The following table summarizes information or features used in previous work to identify and prioritize possible landing sites or zones. In some cases, risk associated with the path returned from the flight planner is used for landing zone prioritization. Specific aircraft performance characteristics are not included at this stage to encourage broad consideration of data for all use cases, although the second column suggests aircraft types primarily considered by the cited references. In practice vehicle performance would impact which features are the most important discriminators.

General aviation and UAS emergency landing planners can store select static data onboard but need access to dynamic attribute data in as close to real time as possible. While terrain and roads may not change, occupancy attributes can dramatically change. For example, a football stadium may be seldom used off-season, but over 100,000 people may be present during a single game. Information services targeting general aviation and UAS user bases could be augmented with relevant data from other sources to help pilots or automation to find suitable emergency landing areas. In case of UAS, such information might substantially improve situational awareness, whereas now responses to anomalies such as component failures or lost link (for UAS) elicit simple response such as fly home, fly to a waypoint or flight-terminate $\frac{13}{13}$ Our paper proposes the use of mobile phone usage databases, a non-traditional information sources to aviation, to estimate occupancy enabling more informed safe landing zone identification and prioritization decisions.

\section{Site Occupation and Population Density from Mobile Phone Databases}

\section{III.A. Overview}

Mobile phones have been almost universally adopted with approximately 6.8 billion subscribers worldwide.14 All those phones can be seen as a sensor network with potential to provide location data ${ }^{a}$ One of the simplest and most explored datasets generated from mobile phones are Call Detail Reports (CDRs) $\frac{15}{n}$ Mobile carriers generate one report each time a mobile phone makes a call or a transaction initially for billing purposes. Those reports basically consist of the caller ID, recipient ID, call time and duration. Moreover, they include information regarding the cell tower used for the caller / recipient. This information can be processed to estimate the geographic position of the phone during the call. When multiple towers hearable from a mobile phone, positioning techniques such as OTDOA (Observed Time Difference of Arrival) can also be used to

\footnotetext{
${ }^{a}$ While users may turn off GPS to save power and may elect not to share their location broadly, the cellular provider can
} still correlate phone with approximate location based on cell tower connectivity. 
Table 1. Data Sources Used in Previous Emergency Landing Site Selection and Flight Planning Work

\begin{tabular}{|c|c|c|}
\hline Reference & Aircraft Type & Used Features (Provider) \\
\hline 1 & Manned Aircraft & $\begin{array}{l}\text { - Road segment dimensions (database) } \\
\text { - Road segment speed limit (database) } \\
\text { - Weather, wind (aviation data) }\end{array}$ \\
\hline 3 & UAS & $\begin{array}{l}\text { - Obstacle and man-made structures free area (sensors) } \\
\text { - Slope (database) } \\
\text { - Surface classification (sensors) } \\
\text { - Weather: wind (aviation data) }\end{array}$ \\
\hline 4 & UAS & $\begin{array}{l}\text { - Obstacle-free area (sensors + database) } \\
\text { - Terrain classification (sensors }+ \text { database) } \\
\text { - Distance from man-made objects (database) } \\
\text { - Surface type, roughness (sensors) }\end{array}$ \\
\hline 5 & UAS & $\begin{array}{l}\text { - Population density (database) } \\
\text { - Terrain slope + classification (database) } \\
\text { - Distance from man-made objects (database) }\end{array}$ \\
\hline 6 & Manned Aircraft & $\begin{array}{l}\text { - Runway dimensions and characteristics (database) } \\
\text { - Airport facilities (database) } \\
\text { - Weather: wind (aviation data) }\end{array}$ \\
\hline 7 & Manned Aircraft & $\begin{array}{l}\text { - Weather: wind, severity, ceiling, visibility (aviation data) } \\
\text { - Runway dimensions (database) } \\
\text { - Airport facilities (database) }\end{array}$ \\
\hline
\end{tabular}

improve cell tower geolocation $[16] 17$

Collections of CDR reports for a geographic region over a period of time opens exciting new possibilities for data mining and usage. Examples applications include analysis of mobility patterns, modeling social interactions, usage of city structures, epidemic spreading, and prediction of socio-economic indicators and outcomes. ${ }^{14}$ Ref. 15 presents different examples of how CDR data might be used for humanitarian development action.

Estimation of population density is another interesting application of CDRs. Ref. 18 develops connections between the underlying population distribution obtained from census estimates with telecommunications data. While some are interested strictly in estimating and analyzing static distributions, other applications can substantially benefit from dynamic distribution estimation and analysis. Ref. 19 provides a detailed discussion of cell phone localization in real-time and presents examples where CDRs are used to show population concentrations during major events such as concerts.

\section{III.B. Database Description}

While companies worldwide are collecting and processing large-scale data for internal purposes, many of these databases are not openly available due to privacy and proprietary data concerns. Cellular phone service providers are particularly sensitive to data sharing for these reasons.

Companies sporadically open data sets to support research and education challenges / competitions of mutual benefit to company and participants. Example of such challenges are Orange's "Data for Development (D4D)" and the "Telecom Italia Big Data Challenge", 14 The latter competition's 2014 data became an open database in December $2014^{20}$ providing a rich source of data to analyze in this paper.

The full open database contains information for two Italian areas, the Milano urban area and the Trentino territory. In addition to cellular usage data, it contains time stamped data such as weather, precipitation, air quality, social media data, news and, in the case of Trentino territory, electrical current usage. In this initial investigation of mobile phone data usage for aviation, this paper uses only the telecommunication activity (SMS, Call, Internet) datasets for the city of Milano collected over the month of December 2013. The entire open database includes information from November 1st, 2013 until January 1st, 2014 and requires

$$
5 \text { of } 11
$$


approximately $5 \mathrm{~GB}$ of storage space in a compressed format.

The database is generated by aggregating data originating from CDRs. Spatial and temporal aggregation combined with units of measure blurring are used for privacy and proprietary reasons. $\frac{18}{1}$ Database entries are summarized below ${ }^{20}$ with symbol associations later referenced by this paper.

- Location - $g$ - grid cell number. Data is provided for each of 10,000 square cells covering the Milano region. Each cell has size $235 m \times 235 m$. Grid geographical locations are available in a separate database defining each square grid element by its four vertex or corner coordinates;

- Time Interval - $t$ - A time stamp in Unix time marking the beginning of a 10 minutes period over which the reported data was collected;

- Country code - $c$ - Country of origin for the phone receiving/sending the communication (depends on other fields);

- SMS-In - $\phi_{1}$ - Activity in terms of received Short Message Service (SMS) inside the grid tile $g$, during the time interval $t$ and sent from the nation identified by the country code $c$;

- SMS-Out - $\phi_{2}$ - Activity in terms of sent SMS inside the grid tile $g$, during the time interval $t$ and received by the nation identified by the country code $c$;

- Call-In - $\phi_{3}$ - Activity in terms of received calls inside the grid tile $g$, during the time interval $t$ and issued from the nation identified by the country code $c$;

- Call-Out - $\phi_{4}$ - Activity in terms of issued calls inside the grid tile $g$, during the time interval $t$ and received by the nation identified by the country code $c$;

- Internet - $\phi_{5}$ - Activity in terms of performed internet traffic inside the grid tile $g$, during the time interval $t$ and by the nation of the users performing the connection identified by the country code $c$.

The original CDRs used to compute the $\phi_{i}$ are generated every time a phone receives or sends an SMS $\left(\phi_{1}\right.$ and $\left.\phi_{2}\right)$, every time a phone receives or issues a call $\left(\phi_{3}\right.$ and $\left.\phi_{4}\right)$, and every time a user starts or stops an internet connection plus every time 15 minutes or $5 \mathrm{MB}$ of usage occurs since the last CDR $\left(\phi_{5}\right) ! 20$

\section{III.C. Database Processing}

After decompression, the first database processing step was aggregation of datasets over all country codes since CDR-based grid occupancy estimates should not be biased by country of information or user origin or destination. Aggregation can be summarized as the following computation:

$$
\phi_{i}(g, t)=\sum_{c=1}^{n_{c}} \phi_{i}(g, t, c)
$$

where $g$ is grid identifier, $t$ is the time interval, $c$ represents the set of all countries for which one or more CDR reports was generated for grid $g$ over time interval $t$, and $n_{c}$ is the total number of country codes. To reduce data storage and transmission overhead, the database omits $\phi_{i}(g, t, c)$ for each case where no data exchange was performed during one of the considered time periods, i.e. $\phi_{i}(g, t, c)=0$. The aggregation step includes back a line to each $(g, t)$ pair that was omitted. This processing step aggregates CDR reports into a single data file with 1440000 lines of 7 entries each for each 24-hour day.

Because numerical entries in the open database are blurred to address privacy and proprietary data concerns ${ }^{b}$ the only information available is that the provided data respects trends, e.g., a higher SMSIn number indicates a higher number of received SMS. Moreover, while the scale of SMS and calls are comparable, internet access scales differently ${ }^{20}$ To plot grid occupancy estimates from telecommunication exchanges as a heat map, the maximum $\phi_{i_{\max }}$, minimum $\phi_{i_{\min }}$ and average $\phi_{i_{\text {avg }}}$ value for each grid or field across the entire database were computed and recorded. For example, $\phi_{i_{a v g}}$ is computed from:

$$
\phi_{i_{a v g}}=\frac{\sum_{g=1}^{n_{g}} \sum_{t=1}^{n_{t}} \phi_{i}(g, t, c)}{n_{g} n_{t}}
$$

\footnotetext{
${ }^{\mathrm{b}}$ An aviation data provider would be better able to negotiate with cellular service providers to ensure accurate (un-blurred) data is represented while respecting privacy and proprietary concerns.
} 
where $n_{g}$ and $n_{t}$ are respectively the total number of grid cells and time intervals. During processing it was observed that $\phi_{i}$ value intervals are too large to support linear interpolation between minimum and maximum value for each cell. Instead, a value of 40 times the overall average of the field was set as a maximum or saturation limit used both for interpolation and clipping of outlier values. Mathematically, the heat maps are created with the following modified features $\bar{\phi}_{i}$ :

$$
\bar{\phi}_{i}(g, t)=\min \left(\frac{\phi_{i}(g, t)}{40 \phi_{i_{a v g}}}, 1\right)
$$

This value was considered adequate to generate heat maps for each $(g, t)$ pair. Later those images were grouped in animations that provide greater insight about the data.

Ultimately, the purpose of CDR data processing is to populate metrics associated with grid occupancy indicated by mobile phone use historical and possibly real-time data trends. Such metrics can be used in emergency landing site selection and in turn emergency or adaptive flight planner 10 An ideal metric would number of people currently occupying any emergency landing grids $g$ the aircraft might be expected to traverse on low final approach, touchdown, and deceleration to a full stop. The goal is thus to process the mobile phone CDR database to estimate population per grid or at least provide a statistical approximation of this population metric. For a real-time estimate, the metric used for plotting the heat-map can be considered a first approach, however, there is also the need to provide a offline computed metric based in historical data that could be loaded as an on-board database.

As an initial estimate, data for each grid was averaged over all time intervals $t$ to generate statistics. However, this simple average was not considered sufficient due to the high variation of mobile network data transfer with time of day. For example the overall number of calls is greater during the day then at night, so even if the same occupancy exists during night and day, daytime figures yield higher usage values. This work tested a simple normalization scheme to combine data over each grid $g$ for each time interval $t$. This normalization $\hat{\phi}_{i}$ is performed by scaling the feature value by the maximum and minimum feature value at that time stamp over all grids. This scaling constrains $\hat{\phi}_{i}$ values to fall between 0 and 1 for each time stamp:

$$
\hat{\phi}_{i}(g, t)=\frac{\phi_{i}(g, t)-\min _{g} \phi_{i}(g, t)}{\max _{g} \phi_{i}(g, t)-\min _{g} \phi_{i}(g, t)}
$$

To estimate overall values $\widetilde{\phi}(g)$, the $\hat{\phi}_{i}$ values were summed and normalized by total data collection time $t$ to estimate average, maximum and minimum time-aggregated feature values:

$$
\widetilde{\phi}_{i, a v g}(g)=\frac{\sum_{t=1}^{n_{t}} \hat{\phi}_{i}(g, t)}{n_{t}} \quad \widetilde{\phi}_{i_{\max }}(g)=\max _{t} \hat{\phi}_{i}(g, t) \quad \widetilde{\phi}_{i_{\min }}(g)=\min _{t} \hat{\phi}_{i}(g, t)
$$

\section{Results and Discussion}

Figure 2 presents a typical heat map constructed with database feature Call-Out $\left(\phi_{4}(g, t)\right)$ with local time $t=10 \mathrm{am}$ presented in the figure as an image overlaid with GoogleMaps. This time shows intense activity commensurate with weekday business hours. The greatest intensities highlight Downtown, Central Rail Station and Linate Airport. Note that the available database region is larger then that presented in Figure 2. but this paper's results are restricted to the depicted window to take full advantage of GoogleMap labels.

The differences between Figure 2 and both graphs shown in Figure 3 reflect the differences between weekday, weekend and night time periods on the volume of mobile communication activities. This is the first observed limitation from using this data for occupancy measurement. During nighttime hours people reduce their mobile use, but a significant fraction of these people remain in the city, even if they "disappear" with respect to the CDRs. These changes suggest that statistical data compiled from other sources and zoning/building maps would be needed to supplement CDR to estimate grid occupancy at least during off-peak (nighttime) hours.

On the other hand, Figure 4 illustrates one of the main potential benefits of the CDR database: its ability to estimate real-time grid occupancies that can significantly differ from statistical (expected) values. On December 22th, 2013, a famous rivalry soccer match Internazionale vs. Milan event took place. Network

activity on this date is presented in Figure 4. Regions of high activity were noted before, during the half time interval (not shown), and after the game. During the game, however, the region does not light up 


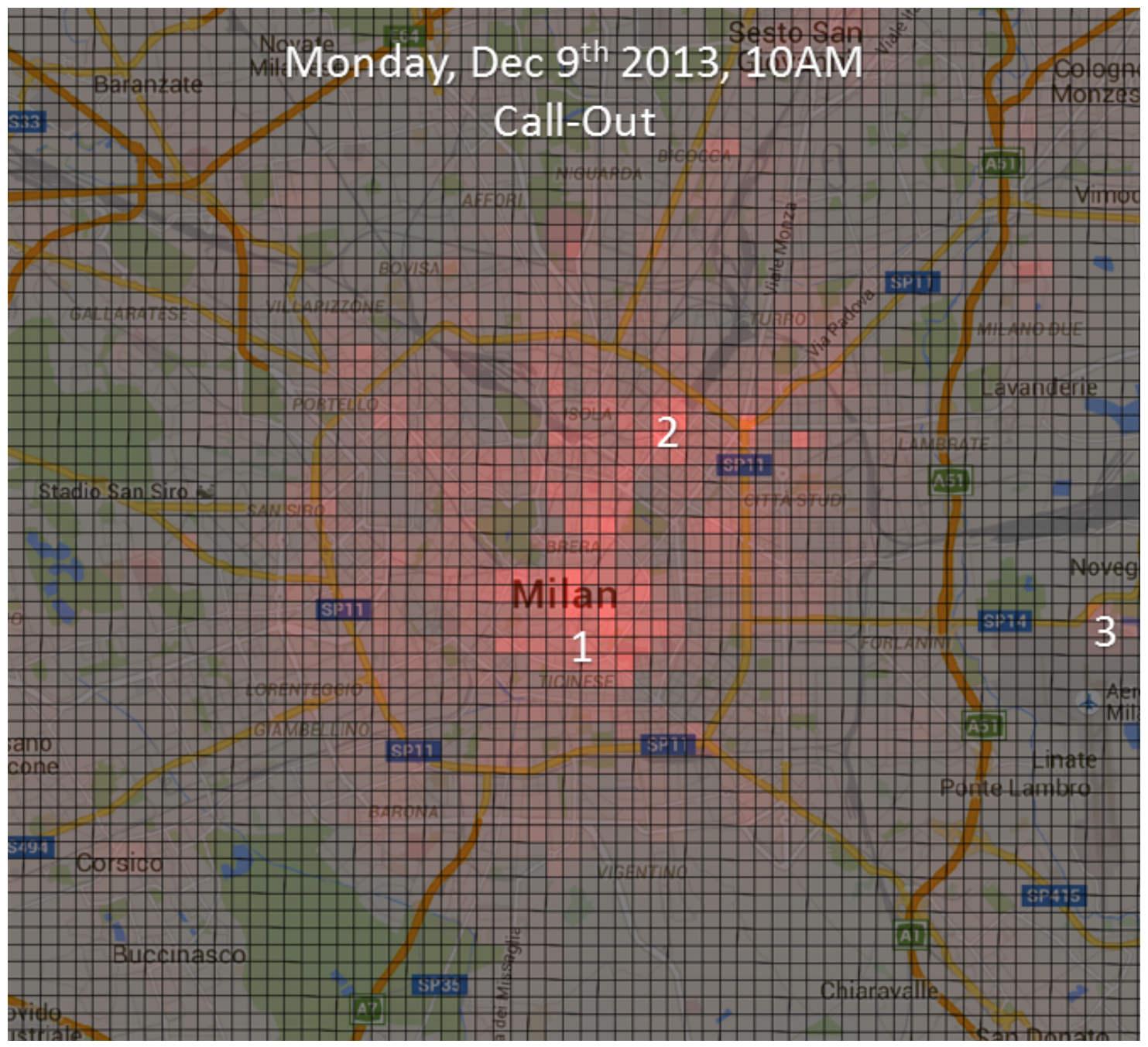

Figure 2. Typical business day activity seen the database. Numbers indicate: 1 - Downtown, 2 - Central Rail Station, 3 - Linate Airport
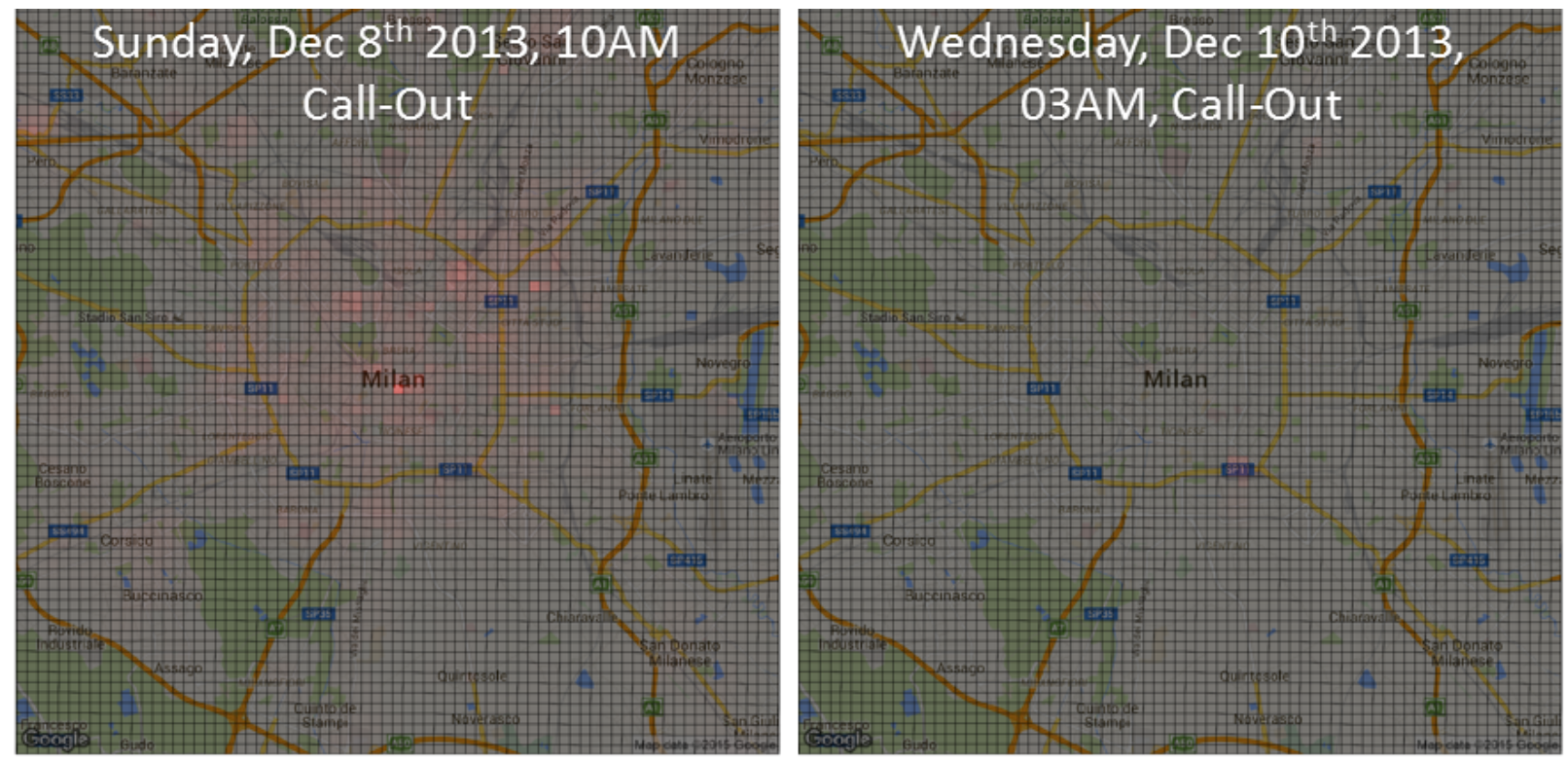

Figure 3. Low activities related to weekend or late night 
significantly even though a huge concentration of people are presented in the stadium. High usage profiles would allow an emergency landing aircraft to recognize and avoid this populated area despite a soccer field normally holding promise as a relatively safe off-runway landing site. However, matching of CDR data to event schedule data will likely be needed to ensure low-CDR periods during the event are still recognized as high occupancy grids by an emergency landing planner.
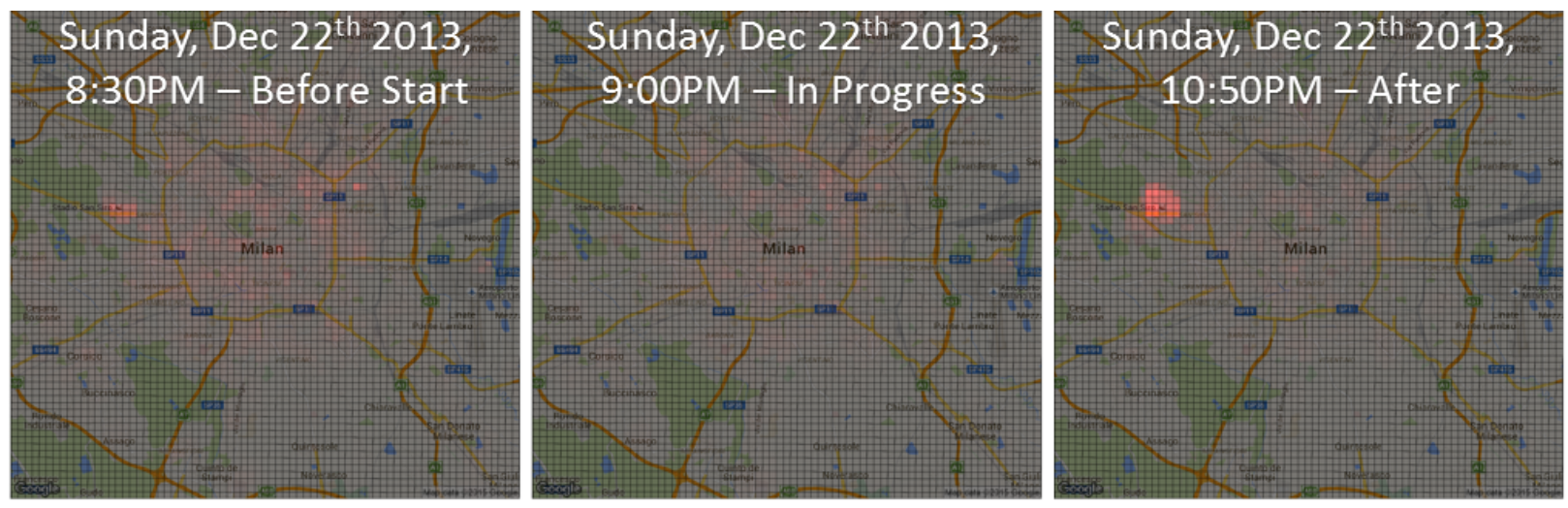

Figure 4. Evolution of Activities Due to Soccer Match
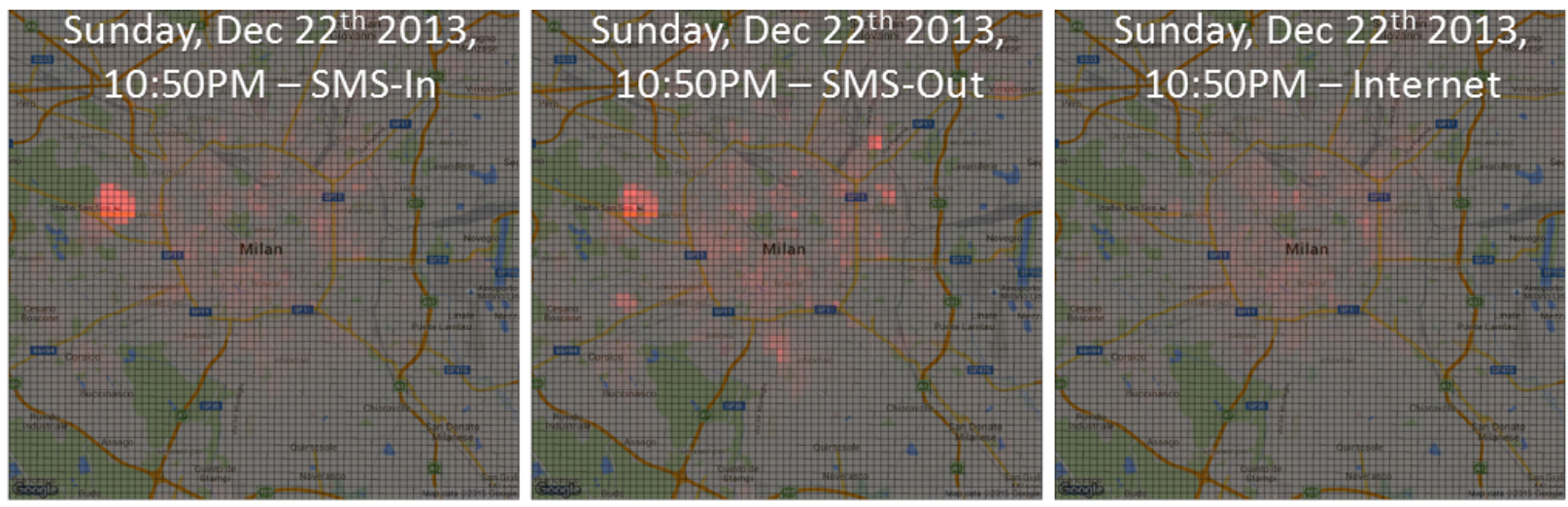

Figure 5. Comparison of Soccer Match Activities in Different Features

The above results only refer to Call-out feature $\phi_{4}$. This option follows Ref. 18 which reports that the Call-out feature gave the highest correlation with population data from traditional (census) databases. Figure 5 shows heat map trends for other CDR features over the same after-match time interval previously presented for Call-out in Figure 4. Note that SMS-In and SMS-Out clearly also show higher activity around the stadium areas. Call-In data is similar to Call-Out data thus not shown here. Internet activity, however, shows a significantly different map, indicating that internet CDR reports may not be a useful indicator of grid occupancy. Additional research however is needed before a definite conclusion could be made about its use.

Figure 6 shows sample compiled data results $\widetilde{\phi}_{4_{\max }}$ and $\widetilde{\phi}_{4_{a v g}}$ that might be utilized in landing site selection exclusively or in combination. Observe that the left map presents several points where one particular time interval was most active. The overall means are presented in the right plot. Heat values are generally low which indicates that no single region has near-maximum mobile activity throughout the data collection period.

These metrics can be used in the following way. Regions with high values of $\widetilde{\phi}_{4, a v g}$ should be avoided since occupancy is likely to be high most of the time. Areas with low values of both $\widetilde{\phi}_{4, \max }$ and $\widetilde{\phi}_{4, \text { avg }}$ may be desirable landing sites since low occupancy is indicated over most times. Finally, areas with low average $\widetilde{\phi}_{4, \text { avg }}$ but high maximum value $\widetilde{\phi}_{4, \max }$ represent areas subject to CDR-based occupancy variation suggesting the need for additional data.

The simple data processing procedure described above illustrates how CDR data might be used to improve landing site selection decisions, but this data use is only a first step. Aggregating data by time of the 

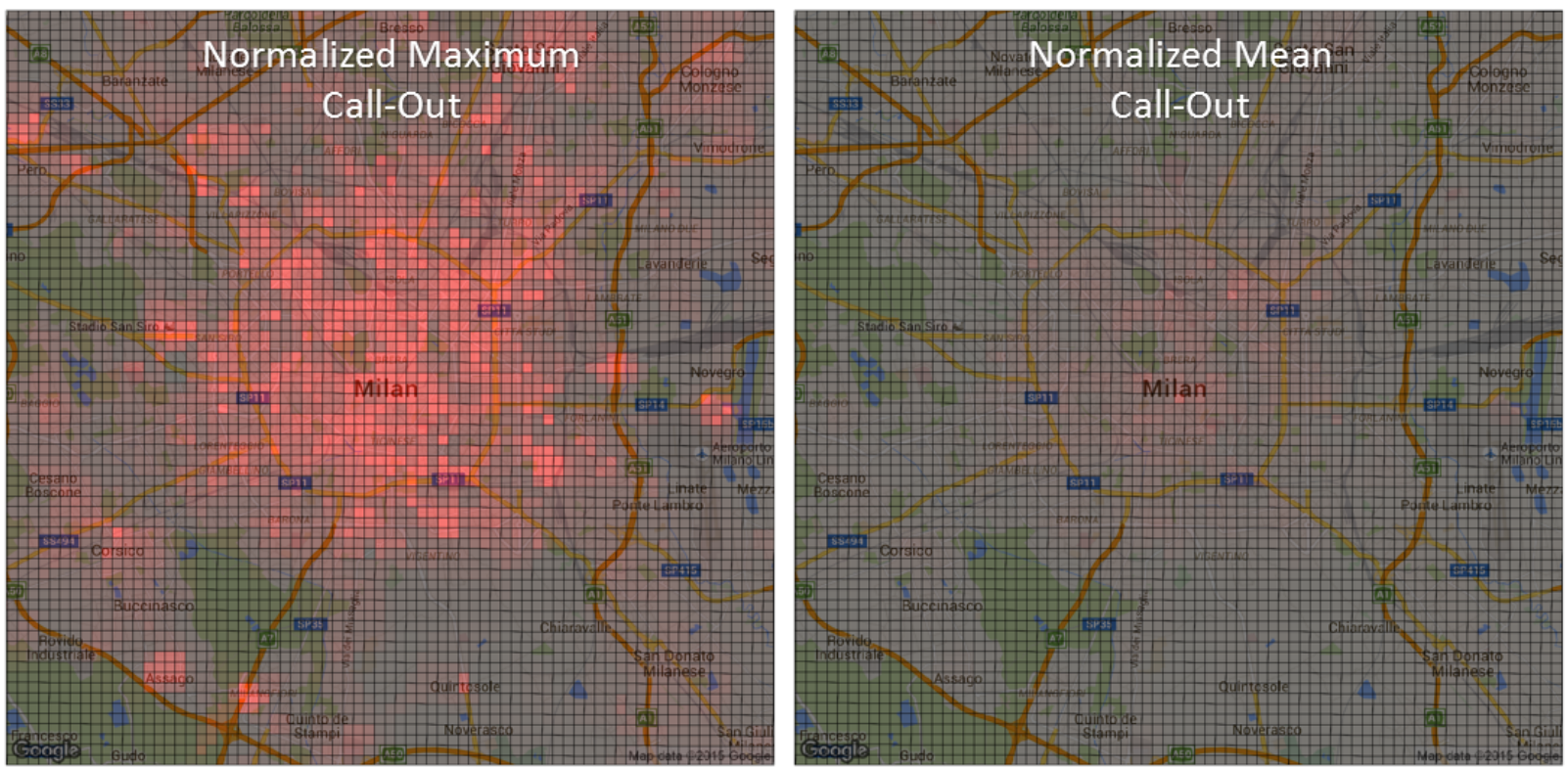

Figure 6. Normalized Maximum and Average Call-Out Values for Each Grid over all Time Intervals

day might be helpful, and linking data to user IDs would also better support population movement trends. However, such augmentations are more likely to result in privacy violations thus must be performed carefully and likely not through open data sharing.

Mobile phone CDR databases will be most effective when used in conjunction with other databases. The first and possibly most desirable combination would be to combine CDR data with terrain or land use data. Initial candidates for landing sites could be selected and then the CDR database could provide further down-selection or prioritization. This analysis could be performed offline using historical data, with only the final candidates been loaded to the airplane. In real time, the emergency landing site selection system could monitor real-time sensors or poll the cloud for real-time occupancy estimates to prioritize feasible landing sites or provide additional data to the pilot.

While CDR appears to provide useful information regarding events, knowledge about scheduled events can also be obtained from event databases. Ref. 21 provides an example of a database that can be queried online for a specific date and location. Such event databases however, only provide the event schedule without additional data on real-time occupancy within a venue or for travel to/from the venue. Moreover, real-time mobile datasets can inform an emergency landing planner even if a large congregation of people assembles without prior planning or formal event scheduling. For example occupation of public parks and golf courses might be estimated from a cellular usage database. Note that occupancy variation in parks was not substantial in the analyzed Milano database, but that could be influenced by the fact that data was reported during a colder fall/winter month.

\section{Conclusion and Future Work}

Emerging automation aids can assist manned aircraft pilots and unmanned aircraft operators in the highly-demanding task of selecting a forced or emergency landing site. On-board sensor data might be augmented with non-traditional database information that can be loaded to the airplane and also communicated in real-time from a broad array of service providers. This paper first reviewed a suite of data sets with potential to improve landing site decisions. The paper then focused on an initial analysis of a new data source: mobile phone Call Detail Reports (CDRs). By processing a database that is publicly-available, this paper proposed processing steps to generate historical occupancy maps that can ultimately be updated in real-time. A significant limitation of CDR data is that it measures cell use not true occupancy, so periods of quiescence such as nighttime and intense event periods (e.g., during a soccer game) show low mobile communication activity relative to true occupancy levels.

Future work involves additional CDR data processing and CDR fusion with other data sources. CDR 
data combined with terrain and land use maps can capitalize on advantages of each information source. Finally, integration within an Adaptive or Emergency Flight Planning automation aid is needed to provide a complete solution to the emergency landing problem.

\section{Acknowledgments and Disclaimer}

The first author is supported in part by CAPES-Brazil through the Science without Borders program, process number BEX 19130/12-0. This work does not reflect official positions of ANAC-Brazil.

\section{References}

${ }^{1}$ Di Donato, P. F. A. and Atkins, E. M., "An Off-Runway Emergency Landing Aid for a Small Aircraft Experiencing Loss of Thrust," Infotech@ Aerospace Conference at Scitech, AIAA, 2015, doi: 10.2514/6.2015-1798

${ }^{2}$ Gautam, A., Sujit, P., and Saripalli, S., "A survey of autonomous landing techniques for UAVs," 2014 International Conference on Unmanned Aircraft Systems (ICUAS), IEEE, 2014, pp. 1210-1218, doi: 10.1109/ICUAS.2014.6842377.

${ }^{3}$ Mejias, L., Fitzgerald, D. L., Eng, P. C., and Xi, L., "Forced landing technologies for unmanned aerial vehicles: towards safer operations," Aerial Vehicles, edited by T. M. Lam, In-Tech, 2009.

${ }^{4}$ Patterson, T., McClean, S., Morrow, P., Parr, G., and Luo, C., "Timely autonomous identification of UAV safe landing zones," Image and Vision Computing, Vol. 32, No. 9, 2014, pp. 568-578, doi: 10.1016/j.imavis.2014.06.006

$\checkmark 5$ Olson, I. J., Ten Harmsel, A. J., and Atkins, E. M., "Safe landing planning for an energy-constrained multicopter," 2014 International Conference on Unmanned Aircraft Systems (ICUAS), IEEE, 2014, pp. 1225-1235, doi: 10.1109/ICUAS.2014.6842379

${ }^{6}$ Atkins, E. M., Portillo, I. A., and Strube, M. J., "Emergency flight planning applied to total loss of thrust," Journal of Aircraft, Vol. 43, No. 4, 2006, pp. 1205-1216, doi: 10.2514/1.18816

${ }^{7}$ Meuleau, N., Plaunt, C., Smith, D. E., and Smith, T. B., "An Emergency Landing Planner for Damaged Aircraft." Proceedings of the Innovative Applications of Artificial Intelligence (IAAI) Conference, AAAI, 2009.

${ }^{8}$ Kopardekar, P. H., "Unmanned Aerial System (UAS) Traffic Management (UTM): Enabling Low-Altitude Airspace and UAS Operations," Tech. Rep. TM-2014-218299, 2014.

${ }_{9}^{9}$ Aubert, M. C., Üzümcü, S., Hutchins, A. R., and Cummings, M., "Toward the Development of a Low-Altitude Air Traffic Control Paradigm for Networks of Small, Autonomous Unmanned Aerial Vehicles," AIAA Infotech@ Aerospace Conference, Kissimmee, FL, 2015, doi: 10.2514/6.2015-1110

${ }^{10}$ Federal Aviation Administration (FAA), "Advisory Circular 00-63, Use of Cockpit Displays of Digital Weather and Operational Information," Tech. rep., 2014.

${ }^{11}$ Harrington, J., "Weather Services in the NextGen Era," Aviation International News, January 2009, pp. 34-38.

${ }^{12}$ Johnson, C., "Using the Internet of Things to Support Dynamic Risk Assessment in Future Concepts of Operation for Air Traffic Management," Tech. rep., 2015, Submitted, available at: http://www.dcs.gla.ac.uk/ johnson/papers/Safecomp2015/ IoTInnovation.pdf, verified at Dec 13, 2015.

${ }^{13}$ Stansbury, R., Wilson, T., and Tanis, W., "A technology survey of emergency recovery and flight termination systems for uas," AIAA Infotech@ Aerospace Conference and AIAA Unmanned... Unlimited Conference, Seattle, OR, 2009, doi: 10.2514/6.2009-2038

${ }^{14}$ Lepri, B., Antonelli, F., Pianesi, F., and Pentland, A., "Making big data work: smart, sustainable, and safe cities," $E P J$ Data Science, Vol. 4, No. 1, 2015, pp. 1-4, doi: 10.1140/epjds/s13688-015-0050-4

${ }^{15}$ United Nations Global Pulse, "Mobile Phone Network Data for Development," Tech. rep., United Nations Global Pulse, October 2013, http://www.unglobalpulse.org/sites/default/files/Mobile\%20Data\%20for\%20Development\%20Primer_ Oct2013.pdf Accessed Dec 12, 2015.

${ }^{16}$ Sun, G., Chen, J., Guo, W., and Liu, K., "Signal processing techniques in network-aided positioning: a survey of state-of-the-art positioning designs," Signal Processing Magazine, IEEE, Vol. 22, No. 4, 2005, pp. 12-23, doi: 10.1109/MSP.2005.1458273

${ }^{17}$ Rufa, J. R. and Atkins, E. M., "Sensor fusion for unmanned aircraft system navigation in an urban environment," American Control Conference (ACC), 2014, IEEE, 2014, pp. 5313-5318, doi: 10.1109/ACC.2014.6858975

$\checkmark{ }^{18}$ Douglass, R. W., Meyer, D. A., Ram, M., Rideout, D., and Song, D., "High resolution population estimates from telecommunications data," EPJ Data Science, Vol. 4, No. 1, 2015, pp. 1-13, doi: 10.1140/epjds/s13688-015-0040-6

$\checkmark{ }^{19}$ Calabrese, F., Colonna, M., Lovisolo, P., Parata, D., and Ratti, C., "Real-time urban monitoring using cell phones: A case study in Rome," IEEE Transactions on Intelligent Transportation Systems, Vol. 12, No. 1, 2011, pp. 141-151, doi: 10.1109/TITS.2010.2074196

20 "Dandelion API - Open Big Data," https://dandelion.eu/datamine/open-big-data/ Accessed Dec 12, 2015.

21 "Eventful," https://api.eventful.com/ Accessed Dec 13, 2015. 
This article has been cited by:

1. Alec J. Ten Harmsel, Isaac J. Olson, Ella M. Atkins. 2017. Emergency Flight Planning for an Energy-Constrained Multicopter. Journal of Intelligent \& Robotic Systems 85:1, 145-165. [Crossref] 